\title{
An Efficient Preconditioner for Linear Systems Issued From the Finite-Element Method for Scattering Problems
}

\author{
Ronan Perrussel, Laurent Nicolas, and François Musy
}

\begin{abstract}
An efficient preconditioner for systems issued from the finite element discretization of time harmonic Maxwell's equations with absorbing boundary conditions is presented. It is based on the Helmholtz decomposition of the electromagnetic field and its discrete counterpart. It is compared to a classical preconditioner on both simple and realistic problems. Its behavior is also evaluated on meshes showing different characteristics.
\end{abstract}

Index Terms-Electromagnetic fields, finite-element methods, numerical analysis, scattering.

\section{INTRODUCTION}

$\mathbf{E}$ LECTROMAGNETIC scattering problems are classically modeled using time harmonic Maxwell's equations with Silver-Müller conditions [1]. The numerical solution of these equations leads to complex and symmetric matrices. To solve these systems, Krylov subspace methods may be used: BiCGCR [2], symmetric QMR [3], or COCG [4]. Classical preconditioning methods are implemented in order to accelerate the convergence of these iterative algorithms: SSOR, incomplete Cholesky factorization [5], etc. An efficient preconditioner based on the Helmholtz decomposition was previously proposed for simple eddy-current problems [6]. The aim of this paper is to test its efficiency on realistic scattering problems and its robustness on meshes with different characteristics. The problem formulation is first given. The preconditioner based on the Helmholtz decomposition is then described. Numerical results are finally presented.

\section{PROBLEM FoRMULATION}

This work deals with time harmonic Maxwell's equations and Silver-Müller conditions. The following finite element formulation, with the incomplete first order edge elements [7] on the domain $\Omega$ (space $\mathbf{Q}_{h}$ ), can be written (for the electric field $\mathbf{E}$ )

Find $\mathbf{E}$ in $\mathbf{Q}_{h, \Gamma_{d}}$ such that:

$$
\begin{aligned}
& a\left(\mathbf{E}, \mathbf{E}^{\prime}\right)=\mathbf{F}\left(\mathbf{E}^{\prime}\right) \quad \forall \mathbf{E}^{\prime} \in \mathbf{Q}_{h, \Gamma_{d}}, \\
& \text { with } a\left(\mathbf{E}, \mathbf{E}^{\prime}\right)=\int_{\Omega} \frac{1}{\mu} \operatorname{curl} \mathbf{E} \cdot \operatorname{curl} \overline{\mathbf{E}}^{\prime} \\
& +\mathrm{i} \int_{\Gamma_{a}} \frac{1}{\mu}\|\mathbf{k}\|(\mathbf{E} \times \mathbf{n}) \cdot\left(\overline{\mathbf{E}}^{\prime} \times \mathbf{n}\right)-\omega^{2} \int_{\Omega} \tilde{\varepsilon} \mathbf{E} \cdot \overline{\mathbf{E}}^{\prime}
\end{aligned}
$$

Manuscript received July 1, 2003.

R. Perrussel is with the laboratories MAPLY and CEGELY, Ecole Centrale de Lyon, 69134 Ecully Cedex, France (e-mail: ronan.perrussel@ec-lyon.fr).

L. Nicolas is with the laboratory CEGELY, Ecole Centrale de Lyon, 69134 Ecully Cedex, France (e-mail: laurent.nicolas@ec-lyon.fr).

F. Musy is with the laboratory MAPLY, Ecole Centrale de Lyon, 69134 Ecully

Cedex, France (e-mail: francois.musy@ec-lyon.fr).

Digital Object Identifier 10.1109/TMAG.2004.824734 where $\omega$ denotes the angular frequency, $\mathbf{k}$ the wave vector, $\mathbf{n}$ the boundary normal direction, $\tilde{\varepsilon}$ the complex-valued permittivity, $\mu$ the permeability, $\mathbf{F}\left(\mathbf{E}^{\prime}\right)$ the source term (incident plane wave), $\Gamma_{a}$ the absorbing boundary, $\Gamma_{d}$ the perfect electric conductor boundary $\left(\mathbf{E} \times \mathbf{n}=0\right.$ on $\left.\Gamma_{d}\right)$. The formulation space of the problem is defined as

$$
\mathbf{Q}_{h, \Gamma_{d}}=\left\{\mathbf{E}^{\prime} \in \mathbf{Q}_{h} / \mathbf{E}^{\prime} \times \mathbf{n}=0 \text { on } \Gamma_{d}\right\} .
$$

Essential characteristics of this formulation are:

- the kernel of the curl operator $(\{\mathbf{E}, \operatorname{curl}(\mathbf{E})=0\})$ is of infinite dimension;

- the sesquilinear form $a$ is not hermitian;

- the operator's spectrum has eigenvalues with positive and negative real parts, the sesquilinear form $a$ is therefore indefinite.

The linear system $A x=b$ to be solved is complex-valued, symmetric, and indefinite. These characteristics are essential for the choice of solving methods.

\section{AN EFFICIENT PRECONDITIONER}

Classical solving methods are adapted to the operator gradient and deal badly with the kernel of the curl operator. Following the Helmholtz decomposition, the electric field $\mathbf{E}$ or the magnetic field $\mathbf{H}$ can be decomposed into two components [7]

$$
\mathbf{E}=\mathbf{E}_{s} \oplus^{\perp} \operatorname{grad} \phi
$$

where:

- $\oplus^{\perp}$ means the orthogonal sum for the scalar product in the square-integrable functions space;

$\operatorname{grad} \phi$ is a static component with $\phi$ a scalar potential. It belongs to the kernel of the curl operator; it is the orthogonal projection on the kernel;

- $\quad \mathbf{E}_{s}$ is a propagation component called solenoidal component. It is divergence-free, because the decomposition is orthogonal.

The curl operator has a dissymmetric behavior on these components [7]. The decomposition's discrete counterpart in $\mathbf{Q}_{h}$ is of practical importance

$$
\mathbf{E}_{h}=\mathbf{E}_{s, h} \oplus^{\perp} \operatorname{grad} \phi_{h}
$$

where:

- $\quad \mathbf{E}_{h}$ belongs to the first incomplete order edge element space $\mathbf{Q}_{h}$

- $\phi_{h}$ belongs to the first order nodal element space $N_{h}$. 


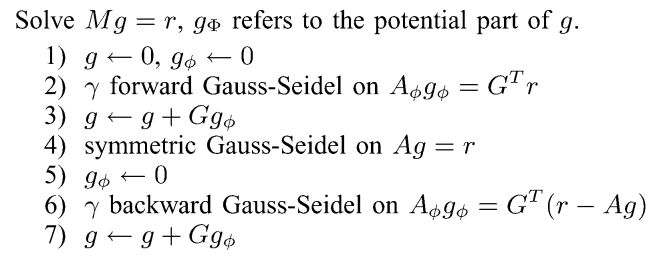

Fig. 1. One iteration of the preconditioning algorithm using the Helmholtz decomposition. Generally $\gamma=1$ or 2 .

Since (1) with $\mathbf{E}=\operatorname{grad} \phi_{h}$ and $\mathbf{E}^{\prime}=\operatorname{grad} \phi_{h}^{\prime}$ gives

$$
\begin{gathered}
a\left(\operatorname{grad} \phi_{h}, \operatorname{grad} \phi_{h}^{\prime}\right)=-\omega^{2} \int_{\Omega} \tilde{\varepsilon} \operatorname{grad} \phi_{h} \cdot \operatorname{grad} \phi_{h}^{\prime} \\
\quad+\mathrm{i} \int_{\Gamma_{a}} \frac{1}{\mu}\|\mathbf{k}\|\left(\operatorname{grad} \phi_{h} \times \mathbf{n}\right) \cdot\left(\operatorname{grad} \phi_{h}^{\prime} \times \mathbf{n}\right)
\end{gathered}
$$

the existence of the scalar potential $\phi_{h}$ enables to consider an auxiliary problem. The SSOR preconditioner has shown to be efficient for this secondary problem (issued from the laplacian operator with specific boundary conditions) [8].

For the implementation, a practical operator to transfer potential representation in the space $N_{h}$ to the field space $\mathbf{Q}_{h}$ is required. Its construction uses the definition of the degrees of freedom (dof) which are: $\int_{e} \operatorname{grad} \phi_{h} \cdot \mathbf{t}$ on each edge $e$ of the mesh for the space $\mathbf{Q}_{h}$, the values on each vertex for the space $N_{h}$. The expression of this operator $G$ for a mesh $T_{h}$ is then issued from the relation for an edge $e$

$$
\underbrace{\int_{\mathbf{x}_{\text {init }}}^{\mathbf{x}_{\text {final }} \operatorname{grad} \phi_{h} \cdot \mathbf{t}}}_{\text {edge element dof }}=\underbrace{\phi_{h}\left(\mathbf{x}_{\mathrm{final}}\right)}_{\text {nodal element dof }}-\underbrace{\phi_{h}\left(\mathbf{x}_{\text {init }}\right)}_{\text {nodal element dof }}
$$

where $\mathbf{x}_{\text {init }}$ and $\mathbf{x}_{\text {final }}$ are the extremities of the edge $e$ and $\mathbf{t}$ the tangential vector to $e$. The global relation $\left\{\operatorname{dof}\left(\operatorname{grad} \phi_{h}\right)\right\}=$ $G\left\{\operatorname{dof}\left(\phi_{h}\right)\right\}$ defines the searched operator $G$ as a sparse matrix with exactly 2 nonzero elements per line: 1 and -1 respectively for the last and first node of each edge.

With this operator, the matrix for the auxiliary problem can be assembled by a Galerkin product: $A_{\Phi}=G^{T} A G$ where $A$ is the edge elements matrix. The numerical cost of this assembly is roughly equivalent to four matrix/vector products with $A$. It can be neglected in comparison with the numerical solving cost (Table II).

Once these elements defined, the algorithm of the preconditioning method can be written (Fig. 1). Note that this method should be incorporated in an iterative solver (COCG, QMR, BiCGCR, etc.). The preconditioning operation simply consists in transforming the residual $r$ into a preconditioned one by solving a linear system $M g=r$ of reduced numerical costs ( $M$ is not necessarily assembled like here).

The cost of one Gauss-Seidel iteration with a matrix is directly linked to its number of nonzeros entries (nnz). The cost of our preconditioner is then a direct function of the nnz of the $A, A_{\phi}$, and $G$ matrices.
TABLE I

NUMBER OF NONZERO ENTRIES FOR EACH MATRIX. $m_{n}$ NB OF NODES

\begin{tabular}{cccc}
\hline \hline matrix & $A$ & $A_{\phi}$ & $G$ \\
\hline Number of non-zeros & $96 m_{n}$ & $13 m_{n}$ & $12 m_{n}$ \\
\hline \hline
\end{tabular}

The approximative nnz in each matrix is given in Table I. It is evaluated with [9] and practical estimations with the test problems

$$
\begin{aligned}
\operatorname{nnz}(A) & =m_{e}+2 *\left(3 * m_{f}\right)+6 * m_{t}, \\
\operatorname{nnz}\left(A_{\phi}\right) & =m_{n}+2 * m_{e}, \\
\operatorname{nnz}(G) & =2 * m_{e}, \\
\text { with } \frac{m_{e}}{m_{n}} & \approx 6, \quad \frac{m_{f}}{m_{n}} \approx 10 \text { and } \frac{m_{t}}{m_{n}} \approx 5 .
\end{aligned}
$$

$m_{n}, m_{e}, m_{f}$, and $m_{t}$ are respectively the number of nodes, edges, faces, and tetrahedral elements in the mesh. It indicates the overcost in terms of memory requirement and supplementary matrix/vector products due to $G$ and $A_{\phi}$. Compared to classical SSOR, it roughly doubles the preconditioning time.

\section{MESH QuALITY}

An intrinsic mesh quality cannot be defined, since it depends on the physical problem being modeled [10]. Different criteria can be considered: good precision, well-conditioned problem, etc.

For the Poisson equation, it was previously shown that the conditioning number of the matrix is linked to the shape of each element and the mesh uniformity [11]. By extension, two parameters to qualify tetrahedron shape and mesh uniformity are used for this formulation.

- The ratio of inscribed $\rho_{\text {ins }}$ over circumscribed $\rho_{\text {circ }}$ sphere radius with a normalizing factor is used to evaluate tetrahedron shape:

$$
\rho=3 \frac{\rho_{\text {ins }}}{\rho_{\text {circ }}} .
$$

This ratio equals 1 if the tetrahedron is regular and decreases to zero if the tetrahedron is fully degenerated.

- The ratio between the largest and the smallest volume of tetrahedra is used to measure the uniformity.

\section{NuMERICAL RESUlTS: EFFICIENCY AND RoBUSTNESS}

Three kinds of comparisons with classical solvers are implemented to test the efficiency of the Helmholtz decomposition preconditioner:

- by increasing the number of degrees of freedom of a given problem;

by analyzing the influence of the mesh quality;

- by computing two realistic problems.

\section{A. Number of Degrees of Freedom}

A $1-\mathrm{GHz}$ plane wave scattered by a 3-D cylinder is studied (Fig. 2). From Fig. 3, it is shown how the number of iterations evolves with the number of degrees of freedom (dof) for the four implemented solving methods: three solvers (COCG, BiCGCR, QMR) with SSOR preconditioning, and a COCG solver with 

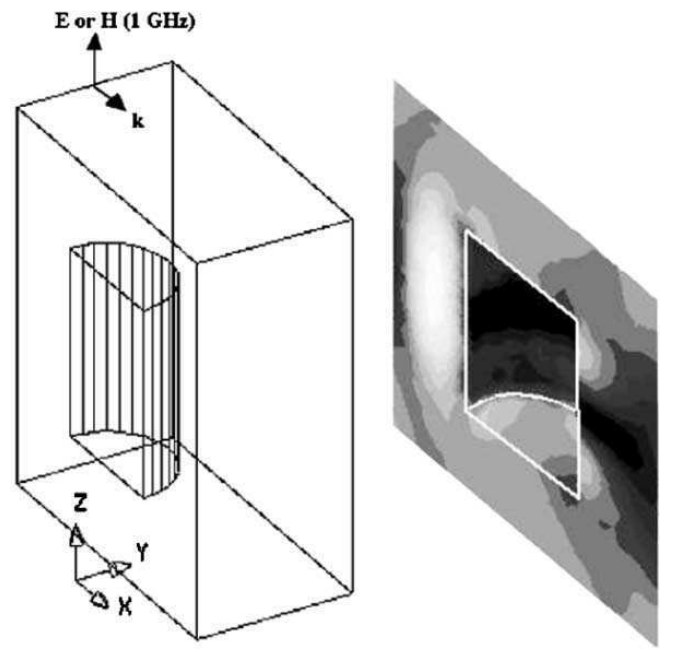

Fig. 2. Incident plane wave on a 3-D cylinder.

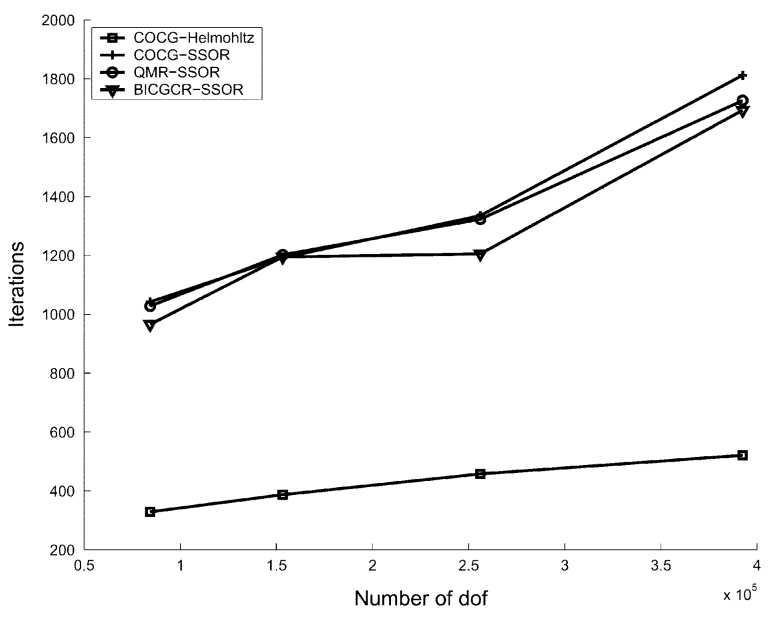

Fig. 3. Number of iterations against the number of dof.

TABLE II

COMPARISON OF CPU TIME (s) FOR THE 3-D CYLINDER

\begin{tabular}{ccccc}
\hline \hline Number of dofs & 84385 & 153293 & 256121 & 392524 \\
\hline QMR - SSOR & 2215 & 5341 & 9807 & 19735 \\
COCG - SSOR & 1862 & 4433 & 8408 & 17175 \\
BiCGCR - SSOR & 2395 & 5214 & 10364 & 22264 \\
COCG - Helmholtz & & & & \\
solving & 1108 & 2312 & 4447 & 7981 \\
assembling preconditioner & 3 & 5 & 8 & 11 \\
\hline \hline
\end{tabular}

the Helmholtz decomposition preconditioner. Table II gives the corresponding CPU times. Here, COCG is the fastest classical solver with SSOR preconditioning. Consequently in the following, only the results with a COCG solver are analyzed. The Helmholtz decomposition preconditioner needs roughly three times fewer iterations and half the CPU time.

\section{B. Quality of the Mesh}

The influence of the quality of the mesh is tested on the 3-D cylinder problem. Mean shape ratio and uniformity of two different meshes are evaluated on this problem (Table III). The
TABLE III

SHAPE RATIO AND UNIFORMity FOR Two MESHES

\begin{tabular}{cccc}
\hline \hline Meshes & $\rho_{\text {mean }}$ & volume ratio & $\frac{\lambda}{h_{\max }}$ \\
\hline $\begin{array}{c}\text { mesh 1 } \\
\text { 463 213 elem. }\end{array}$ & 0.795 & 77.2 & 9.2 \\
\hline $\begin{array}{c}\text { mesh 2 } \\
63 \text { 565 elem. }\end{array}$ & 0.784 & 8682 & 0.95 \\
\hline \hline
\end{tabular}

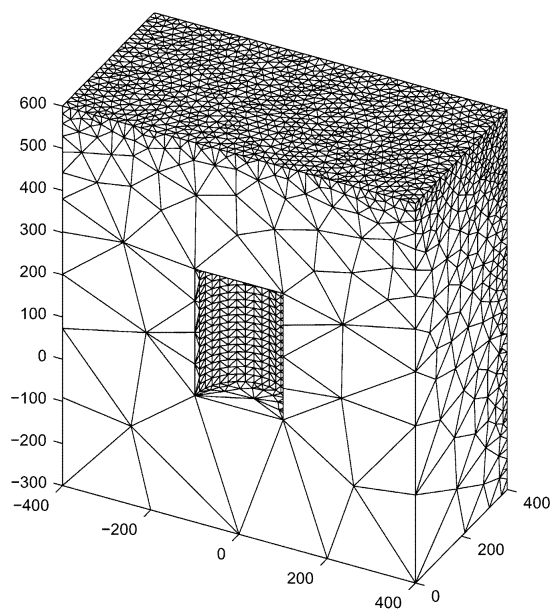

Fig. 4. Lack of uniformity for mesh 2.

TABLE IV

CPU TIME AND ITERATIONS FOR Two MESHES

\begin{tabular}{cccc}
\hline \hline Meshes & & COCG-Helmholtz & COCG-SSOR \\
\hline mesh 1 & iter. & 590 & 1582 \\
557539 dof & CPU (s) & 14690 & 21603 \\
\hline mesh 2 & iter. & 1400 & $>8000$ \\
72229 dof & CPU (s) & 4935 & \\
\hline \hline
\end{tabular}

ratio $\left(\lambda / h_{\max }\right)$ is also given, where $\lambda$ is the wavelength and $h_{\max }$ the length of the longest edge in the mesh.

The mean shape ratio is equivalent for both meshes. The main difference is concerning uniformity: mesh 2 (Fig. 4) is less uniform than mesh 1.

In Table IV, the influence of the quality of the mesh on the convergence is illustrated. The convergence is greatly slowed for mesh 2. The effect is significant even with the Helmholtz decomposition preconditioner. However, it is largely more robust than the SSOR preconditioner, which does not converge after 8000 iterations.

The ratio $\left(\lambda / h_{\max }\right)$ is less than 1 in mesh 2 . However, a mean of 10 nodes per wavelength is necessary to correctly discretize the wave equation. Note that the physical validity of this discretization is doubtful. The influence of this ratio is tested on the mesh 2 by reducing the frequency of the incident wave, which leads to increase the ratio $\left(\lambda / h_{\max }\right)$. Fig. 5 shows the number of iterations as a function of the frequency for both solvers. Table $\mathrm{V}$ presents corresponding CPU times. In the considered frequency band, both solvers converge. Obviously, the Helmholtz decomposition preconditioner performs better than the SSOR preconditioner and is less sensitive to the ratio $\left(\lambda / h_{\max }\right)$. 


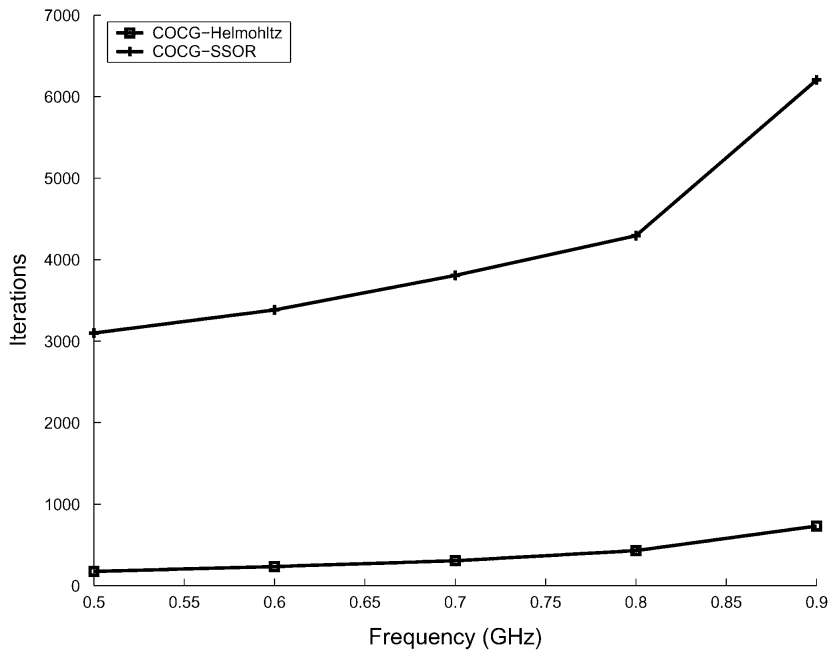

Fig. 5. Number of iterations against the frequency.

TABLE V

CPU Time (s) FunCTION OF FREQUENCIES-MESH 2

\begin{tabular}{cccc}
\hline \hline Frequencies $(\mathrm{GHz})$ & 0.5 & 0.7 & 0.9 \\
$\frac{\lambda}{h_{\max }}$ & 1.9 & 1.36 & 1.06 \\
\hline COCG-SSOR & 5894 & 6425 & 7225 \\
COCG-Helm. & 612 & 816 & 1044 \\
\hline \hline
\end{tabular}
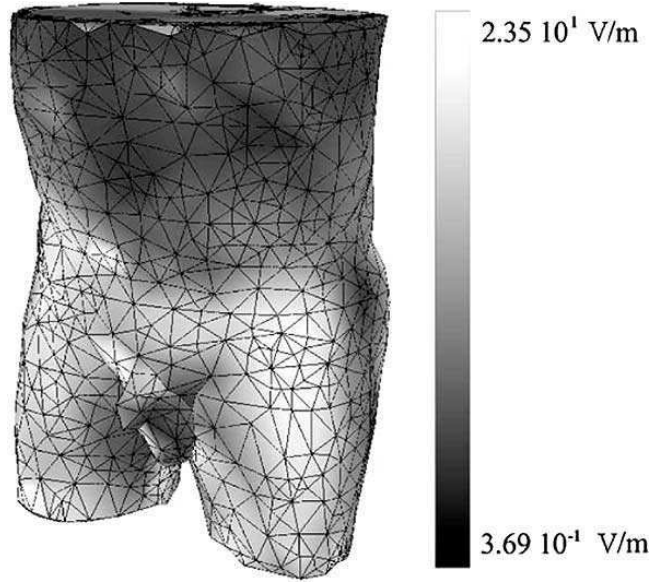

Fig. 6. Hyperthermia RF (27 MHz) for treating deep tumors; magnitude of the electric field.

\section{Realistic Problems}

The efficiency of the Helmholtz decomposition preconditioner is observed on two realistic problems. In the first problem (Fig. 6), the electric field due to a RF source is computed inside a human body during an hyperthermia treatment [12]. The second problem models (Fig. 7) an airplane illuminated by a plane wave [13]. The Helmholtz decomposition preconditioner shows its efficiency in both cases (Table VI), more particularly in the hyperthermia case, for which COCG-SSOR did not converge after 8000 iterations.

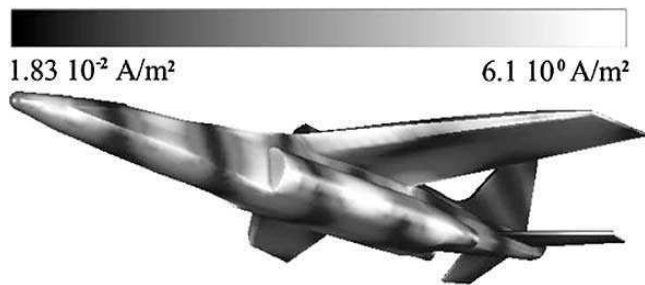

Fig. 7. Illumination of a plane by a $100 \mathrm{MHz}$ plane wave; magnitude of the current density.

TABLE VI

CPU TIME AND ITERATIONS FOR Two REALISTIC PROBLEMS

\begin{tabular}{ccc}
\hline \hline Problems & Hyperthermia & Plane \\
\hline Number of dofs & 202701 & 574151 \\
\hline COCG - SSOR & & \\
Number of iterations & $>8000$ & 2890 \\
CPU time(s) & & 40096 \\
\hline COCG - Helmholtz & & \\
Number of iterations & 474 & 1210 \\
CPU time(s) & 3362 & 26320 \\
\hline \hline
\end{tabular}

\section{CONCLUSION}

A preconditioner based on the Helmholtz decomposition has been developed for scattering problems. This method is efficient because well adapted to the curl operator. The robustness has been tested on a nonuniform mesh. Its efficiency has been evaluated on realistic problems. Furthermore, it is simple to implement and requires only a light overcost.

\section{REFERENCES}

[1] D. Colton and R. Kress, Inverse Acoustic and Electromagnetic Scattering Theory. Berlin, Germany: Springer-Verlag, 1992.

[2] M. Clemens and T. Weiland, "Iterative methods for the solution of very large complex symmetric linear systems of equations in electrodynamics," IEEE Trans. Magn., vol. 34, pp. 3335-3338, Sept. 1998.

[3] R. W. Freund, G. H. Golub, and N. M. Nachtigal, "Iterative solution of linear systems," Acta Numerica, vol. 1, pp. 57-100, 1992.

[4] H. A. van der Vorst and J. B. M. Melissen, "A Petrov-Galerkin type method for solving $A x=b$ where $A$ is symmetric complex," IEEE Trans. Magn., vol. 26, pp. 706-708, Mar. 1990.

[5] G. Meurant, Computer Solution of Large Linear Systems. Amsterdam, The Netherlands: Elsevier, 1999.

[6] R. Beck, "Algebraic multigrid by components splitting for edge elements on simplicial triangulations," Preprint SC 99-40, ZIB, Dec. 1999.

[7] R. Hiptmair, "Multigrid method for Maxwell's equations," SIAM J. Numer. Anal., vol. 36, pp. 204-225, 1999.

[8] O. Axelsson and A. V. Barker, Finite Element Solution of Boundary-Value Problems, Theory and Computation. New York: Academic, 1984.

[9] P. R. Kotiuga, "Essential arithmetic for evaluating three dimensional vector finite element interpolation schemes," IEEE Trans. Magn., vol. 27, pp. 5208-5210, Nov. 1991.

[10] P. J. Frey and P.-L. George, Maillages-Applications aux Elements Finis. Paris, France: Hermes, 1999.

[11] J. R. Shewchuk, "What is a good linear element? Interpolation, conditioning, and quality measures," in 11th Int. Meshing Roundtable, 2002.

[12] N. Siauve, L. Nicolas, C. Vollaire, and C. Marchal, "3D modeling of electromagnetic fields in local hyperthermia," Eur. Phys. J. AP, vol. 21, pp. 243-250, 2003.

[13] C. Vollaire and L. Nicolas, "Implementation of a finite element and absorbing boundary conditions package on a parallel shared memory computer," IEEE Trans. Magn., vol. 34, pp. 3343-3346, Sept. 1998. 